\title{
Short paper:
}

The classical mean negative asynchrony in sensorimotor synchronization is not universal in humans. A cross-cultural study.

\author{
Julien Lagarde \\ Julien.lagarde@umontpellier.fr
}

\author{
Euromov DHM \\ University Montpellier, France
}

Acknowledgements: The author wish to thank Simon Bastide and Anthony Laurens for help in data collection. Writing of this manuscript was supported by the European Project ENTIMEMENT, H2020-FETPROACT-2018, Grant Number 824160.

\begin{abstract}
The present study examines to what extent cultural background determines sensorimotor synchronization in humans. The direct comparison of Indian and French students, without particular experience in music or dance, or sport, was motivated by the hypothesis that musical exposure to different musical styles causes a variation in basic synchronization to sound function. At first rate limits of this capacity was sought, using a parametric design increasing the sound periodic frequency up to synchronization breakdown. No robust effect was found in that respect. However, another unpredicted change of the so-called negative mean asynchrony was found. Negative mean asynchrony is defined as the anticipation of movement with respect to sound, of about $40 \mathrm{~ms}$. The negative mean asynchrony simply disappears in Indians' participants. This result is very intriguing as negative mean asynchrony was considered ubiquitous for decades, and an invariant hallmark of human timing function. Revision of theoretical modelling of sensorimotor synchronization may be required to account to the found variation. Previous modelling attempts in terms of so-called anticipatory or delayed processes, like the Paillard- Fraisse hypothesis, are likely to be revised. A larger adaptation to the rate of
\end{abstract}


events, like the one proposed by Bard Ermentrout (1991) to account for reduced phase shift in firefly Pteroptyx malaccae, offers some promising modelling avenues.

Acting in a cultural niche brings about repeated interaction with temporal patterns of events. As early as mother- infant vocal interactions (Gratier, 2003), rhythmic behaviours display universals and cross-cultural specifics (Cameron et al., 20015; Jacoby \& McDermott, 2017). Here we examine how culture shape rhythm skills and specifically determine elementary synchronization. We used the most elementary expression of human timing function involving movement: The sensorimotor synchronization paradigm. This paradigm has been particularly instrumental in the study of basic human function, with a rare blend of empirical (See Repp, 2005 and Repp \& Su, 2013, for reviews; and Repp, 2021 for some historical background), and theoretical- modelling studies (Bose et al., 2019; Chen et al., 1997; Gonzalez, et al., 2019; Hayashi et al., 2016; Kelso et al., 1990; Schöner, 2002). Some of the dynamical and stochastic properties of sensorimotor synchronization are considered, depending on theoretical assumptions, as belonging to self-organized entrainment phenomena, or based upon explicitly anticipatory processes involving error correction using an interval memory of time intervals. Those properties, and their assumed determiners, are considered to a large extent time-invariant for a given individual, however some flexibility with learning and expertise is found in synchronization tasks (Jantzen et al. 2001; Repp, 2010).

We made the hypothesis that culture determine to some extent the way people synchronize their movement to simple sound sequences. As a start here we compared synchronization behaviours of Indians and Frenchs participants. Data collected, including 15 French and 15 Indian participants, show interesting differences in the way to synchronize to a simple beat. For definitions and analysis, this study uses the theoretical framework of coordination dynamics. The general model is a non-linear model of a self-sustained oscillator (1.h.s.), forced by a periodic function, representing the heard rhythmic sound, and random noise (r.h.s.):

$\ddot{x}+\dot{x}^{3}-\dot{x}+\dot{x} \cdot x^{2}+\omega 0 \cdot x=\varepsilon \cdot \sin (\omega \cdot t)+\sqrt{Q} \cdot \xi t \quad E q .1$

$\omega 0$ controls for the intrinsic rhythm of the oscillator

$x, \dot{x}, \ddot{x}$ represent position, speed and acceleration respectively

$\varepsilon$ controls the strength of the forcing (think of sound intensity of attention for instance)

$\omega$ stands for the pulsation (frequency or "rate") of the external forcing by sound 
$\sqrt{Q} . \xi t$ accounts for random noise in biological system according to a Langevin framework

For the mathematical investigation of models of the type shown in Eq.1, it is classical to study a reduction to a phase equation, and to a discrete map. It is well known that this model of synchronization obeys the theory of Arnold's tongues (Kelso \& DeGuzman, 1988), providing theoretically grounded determiners of synchronization. From this equation relative phase dynamics can be defined, including the bistable dynamics of two stable attractors, synchronization and syncopation, resp. in phase and antiphase, empirically described in details with a parametric variation of rate of sound sequence (Kelso et al., 1990; Eq. 2): $\dot{\phi}=\Delta \omega+$ a. $\sin \phi-$ b. $\sin 2 \phi+\sqrt{Q} \cdot \xi t \quad E q .2$

$\dot{\phi}$ is the time derivative of the relative phase

$\phi$ is the relative phase (phase difference between sound sequence and movement position) $\Delta \omega$ accounts for the difference between actual frequency of the sound and preferred frequency of movement execution

$a$ and $b$ are real numbers controlling the stability of synchronization and syncopation $\sqrt{Q} . \xi$ t stands for biological noise

This model in Eq. (2) is a reduction of the forced oscillator model, it enables to focus on dynamics of synchronization with 1:1 frequency ratio, and accounts for a variety of behaviours when parameters are varied, notably the onset and loss of synchronization or syncopation, and transition between those two patterns. Its study and use, or variations of it like the classical Adler equation, has been pursued in a wide range of disciplines. It is noteworthy though not addressed here that the forced oscillator model accounts also for synchronization at other ratios of frequencies than $1: 1$.

Here we study the in phase synchronization pattern, therefore equation Eq. 2 can be linearized around the corresponding stable fixed point to obtain meaningful observables, notably concerning stability, and their relations (statistics from the Gaussian distribution corresponding to the well-known Ornstein- Uhlenbeck process, or AR1 process, including mean and variance, but also relaxation time, autocorrelation, and power spectrum; See Schöner \& Kelso, 1986; Van Kampen, 1992). 
We ran an experiment to examine rate limits of synchronization and mean phase shift of synchronization in stationary time series. The latter is equivalent to the negative mean asynchrony (NMA), known to exhibit "the reported tendency of humans to tap on average prior to tone onsets" (from Bose et al. 2019).

\section{Participants}

Indians and French participants ( $N=15$ in each group, 11 men and 4 women, age 22 to 45$)$, all students at the university, right handed, recruited in Montpellier, were matched in pairs to control for education, age, and had limited musical, or dance, or sports experience. Indians recruited had left India for France less than 2 years before the experiment, their mother tongue was Indian, their second language English, and they were not fluent in French. Participants gave their informed consent before the experiment.

\section{Task}

The task was to synchronize as best as possible a tap on the table of the index finger with a sound. 3 trials were completed. The frequency of the sound beats $(40 \mathrm{~ms}$; carrier frequency $440 \mathrm{~Hz}$ ) sequence was increased every 15 stimuli by $0.3 \mathrm{~Hz}$. The range of the pacing frequency went from 1 to $6.1 \mathrm{~Hz}$.

Data collection

A goniometer was used to collect the index finger position (metacarpophalangeal angle), connected to an A to D card, also used to collect stimuli. To get a good accuracy for determining the temporal center of each auditory beat we collected all signals at $5 \mathrm{KHz}$. A second PC and the sound D to A card was used to display the stimuli.

\section{Data pre-processing}

Angular positions were down sampled to $500 \mathrm{~Hz}$ and low pass filtered at $30 \mathrm{~Hz}$ with dual pass to negate the phase shift. Stimuli were processed to identify the time of each center of beat, using a low pass (dual pass) filter and local maxima estimation.

\section{Data processing to measure synchronization}

The relative phase between position and beats was estimated taking the value of the Hilbert transform phase of the position at each stimuli onset. Transients (beginning of each plateau) 
were excluded when calculating mean and dispersion of relative phase. The angular mean and dispersion (circular methods) were estimated and are well defined in the sampled stationary behaviours. The mean relative phase, an angle variable, is here somewhat equivalent to the mean time difference, aka mean negative asynchrony (Aschersleben, 2002), classically used in the literature (See Lagarde \& Kelso 2006 for illustration of relations between the two variables). The relative phase fits naturally with the geometry and dynamics of the oscillator model of synchronization. This difference between two angles, in radians, brings some invariance when rate is increased, as one has to (normalize) divide it by the pulsation (rate of rotation on the circle, in radians per second, equivalent to frequency) to get temporal asynchrony in seconds.

\section{Results}

The maximal rates at which French and Indian participants were able to synchronize were comparable (not shown). However, as shown in Figure 1, the histogram of the relative phase between movement and sound is centred onto zero, the classical shift from zero of the mean asynchrony is not observed in the Indians participants. Figure 2 shows the distribution of the relative phase between movement and sound beats, and corresponding dispersion and mean of time series, when rate of metronome was increased. The classical dynamical hallmarks of synchronization, its formation (stable) and breakdown (unstable) are found (Kelso et al., 1990; Lagarde et al., 2006). Two qualitatively distinct behaviours are observed, accounted for by the two main regimes of a model of forced oscillator for 1:1 synchronization. First, at lower rates, a stable pattern of synchronization, the stability of which can vary, and second a loss of stability of this pattern. To avoid a possible confusion, in the second case this corresponds to a (total) loss of stability and not a simple decrease of stability, in other words a breakdown of the synchronization pattern. For rates for which the brain is able to maintain a stable synchronization, time series of relative phase is steady around a mean with a dispersion dependent upon its stability, ii) with increase of rate the synchronization pattern loses its stability (totally, in a qualitative manner so to speak), the relative phase wanders in the $\{-$ pi; pi interval; this happens at different rates for different participants; but mainly around $4 \mathrm{~Hz}$. Please note that transients and stable time series have not been set apart here, thus the ensemble dispersion in a group can be produced by time series corresponding to a decrease of stability or to a loss of stability. 

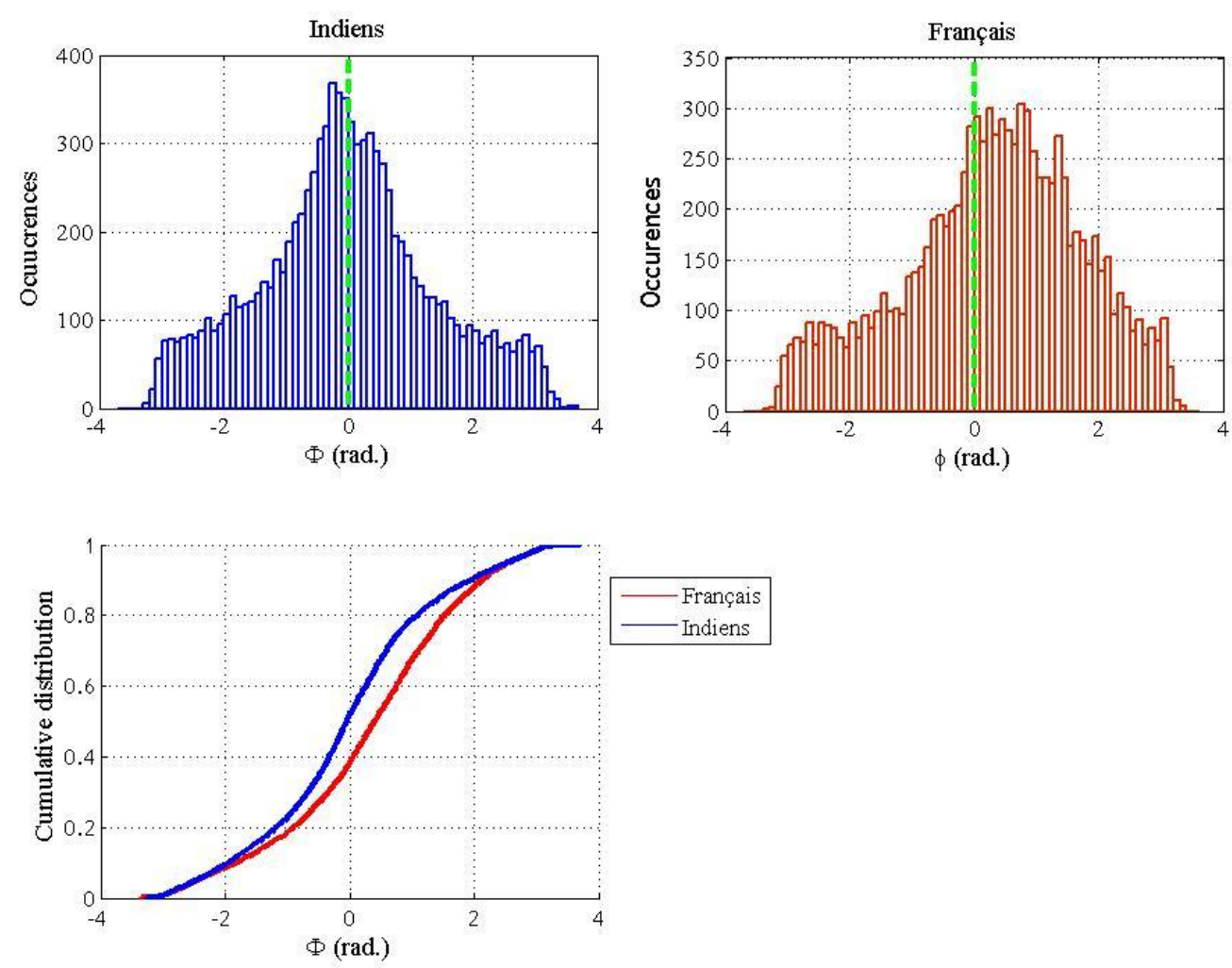

Figure 1. The first task, frequency ranging from $1 \mathrm{~Hz}$ to $6.1 \mathrm{~Hz}$. Histograms of relative phases for all the plateaus for French and Indian participants ( $N=9720$ values; bin size 0.1 radians). The lower panel shows the cumulative distributions; a Kolmogorov-Smirnov test on the maximal difference between cumulative distributions confirms a significant difference between the distributions of the two groups. The distribution of French participants is centred toward positive relative phase, while for the Indians participants the distribution is centred on negative values. Please remember that the sign is reversed relative to usual conventions: Positive correspond to a movement advance in time with respect to the stimuli, which is the classic mean negative asynchrony. 

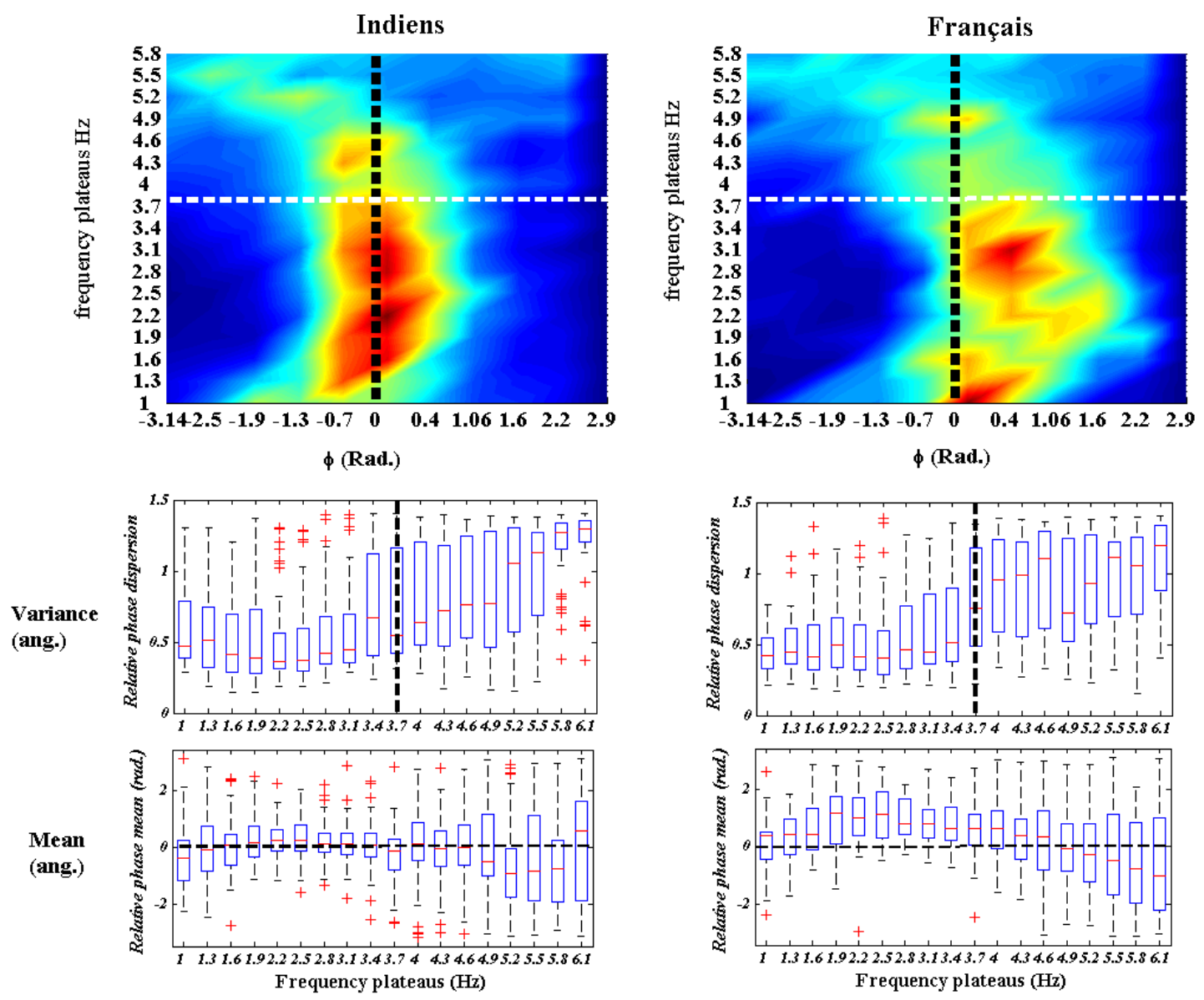

Figure 2 The first task, frequency ranging from $1 \mathrm{~Hz}$ to $6.1 \mathrm{~Hz}$. On the top row the color coded occurrences estimated from histograms of the relative phase as a function of the frequency of the stimuli (Red is high occurrences, blue is rare occurrences). On the middle row, the box plot of the angular dispersion (variance) of the relative phase. The dotted vertical line is added to indicate the increase of dispersion of synchronization with rate at about $3.7 \mathrm{~Hz}$. This increase is mainly due to a loss of (stable) synchronization, however the rate at which this happens varies between participants. On the bottom row, the box plot of the mean of the relative phase. Please remember that the sign is reversed relative to usual conventions: Positive correspond to a movement advance in time with respect to the stimuli, which is the classic mean negative asynchrony.

The color coded histograms and the boxplot of the mean relative phase in Figure 2, show that while French participants displayed the classical NMA (here due to the conventions used 
positive values about 0.5 to 1 radians), the Indians participants synched without displaying the NMA.

\section{Discussion}

Our hypothesis of an effect of cultural origin on a basic synchronization behaviour was not confirmed by the analysis of the rate limit of synchronization. However, to our surprise, the classical negative mean asynchrony was largely affected by the cultural origin of participants. The negative mean asynchrony is among the most frequently reported features of the sensorimotor function corresponding to synchronization to a sound in humans (Repp 2005), and has been interpreted as a caused by universal mechanisms, like neurophysiological delays, aka the Paillard- Fraisse hypothesis, and so called "anticipatory" cognitive processes, driving several modeling attempts until recently (See Bose et al., 2019; Ishida \& Sawada, 2004). It is considered as a ubiquitous feature of this most basic example of timing function in humans (Aschersleben, 2002). Here we found using the elementary tapping task methodology that this negative mean asynchrony is not universal among humans. The variation of rate of stimuli using plateaus showed that difference across a range of rate. This original and unexpected results calls for new modelling (See promising perspectives in Ermentrout, 1991, and in the same vein Savinov et al., 2021), and neuroimaging studies (Jantzen et al., 2004; Nozaradan et al., 2016). Importantly, a simple cross-cultural comparison in elementary sensorimotor behaviour may motivate renewed theoretical assumptions (See Kupferschmidt, 2019). Further modelling and experiments could ask to what extent the behavioural differences observed between the Indians and French synchronization come from sensorimotor adjustments evolving at two time scales, corresponding to period versus phase adjustments- dynamics (Ermentrout, 1991; Savinov et al., 2021). An important question for future studies is whether a unique model can account for the two synchronization behaviours found here, or whether two qualitatively distinct processesmodels are required. One may relatedly attempt to isolate essential aspects of individual's early development, interactions or experience, and cultural factors that determine those differences, and following Jacoby \& McDermott (2017) examine whether such variations widely distribute among humans.

\section{References}


Aschersleben, G. (2002). Temporal control of movements in sensorimotor synchronization. Brain and cognition, 48(1), 66-79.

Aschersleben, G., \& Prinz, W. (1997). Delayed auditory feedback in synchronization. Journal of motor behavior, 29, 35-46.

Bose, A., Byrne, A., \& Rinzel, J. (2019). A neuromechanistic model for rhythmic beat generation. PLoS computational biology, 15(5), e1006450.

Cameron, D. J., Bentley, J., \& Grahn, J. A. (2015). Cross-cultural influences on rhythm processing: reproduction, discrimination, and beat tapping. Frontiers in Psychology, 6, 366.

Chen, Y., Ding, M., \& Kelso, J. S. (1997). Long memory processes (1/f $\alpha$ type) in human coordination. Physical Review Letters, 79(22), 4501.

Ermentrout, B. (1991). An adaptive model for synchrony in the firefly Pteroptyx malaccae. Journal of Mathematical Biology, 29(6), 571-585.

Gratier, M. (2003). Expressive timing and interactional synchrony between mothers and infants: Cultural similarities, cultural differences, and the immigration experience. Cognitive development, $18(4)$, 533-554.

Hayashi, Y., Nasuto, S. J., \& Eberle, H. (2016). Renormalized time scale for anticipating and lagging synchronization. Physical Review E, 93(5), 052229.

Ishida, F., \& Sawada, Y. E. (2004). Human hand moves proactively to the external stimulus: an evolutional strategy for minimizing transient error. Physical review letters, 93(16), 168105.

Jacoby N, McDermott JH (2017). Integer ratio priors on musical rhythm revealed crossculturally by iterated reproduction. Current Biology, 27, 359-370.

Jantzen, K. J., Steinberg, F. L., \& Kelso, J. S. (2004). Brain networks underlying human timing behavior are influenced by prior context. Proceedings of the National Academy of Sciences, $101(17)$, 6815-6820.

Jantzen, K. J., Fuchs, A., Mayville, J. M., Deecke, L., \& Kelso, J. S. (2001). Neuromagnetic activity in alpha and beta bands reflect learning-induced increases in coordinative stability. Clinical Neurophysiology, $112(9)$, 1685-1697.

Kelso JAS, DeGuzman GC (1988) Order in time: how cooperation between the hands informs the design of the brain. In: Haken H (ed.) Neural and Synergetic Computers. Springer, Berlin Heidelberg New York, pp 180-196.

Kelso JAS, DelColle J, Schöner G. (1990). Action-perception as a pattern formation process. In: Jeannerod M (Ed.). Attention and Performance XIII. Hillsdale, NJ: Erlbaum. pp. 139-169. 
Kupferschmidt, K. (2019). Psychologist aims to study diverse minds, not WEIRDos.Science 365 (6449), 110.

Lagarde J, Kelso JAS (2006) Binding of movement, sound and touch: multimodal coordination dynamics. Exp Brain Res. 173, 673-88.

Nozaradan, S., Peretz, I., \& Keller, P. E. (2016). Individual differences in rhythmic cortical entrainment correlate with predictive behavior in sensorimotor synchronization. Scientific Reports, 6(1), 1-12.

Repp, B. H. (2005). Sensorimotor synchronization: a review of the tapping literature. Psychonomic bulletin \& review, 12(6), 969-992.

Repp, B. H. (2010). Sensorimotor synchronization and perception of timing: effects of music training and task experience. Human movement science, 29(2), 200-213.

Repp, B. H. (2012). The legacy of Paul Fraisse: II. Two further studies of sensorimotor synchronization. Psychomusicology: Music, Mind, and Brain, 22(1), 74.

Repp, B.H., Su, Y.H. (2013). Sensorimotor synchronization: a review of recent research (20062012). Psychonomic bulletin \& review, 20(3), 403-452.

Savinov, M., Swigon, D., \& Ermentrout, B. (2021). Synchronization and locking in oscillators with flexible periods. Chaos: An Interdisciplinary Journal of Nonlinear Science, 31(3), 033143. Schöner, G. (2002). Timing, clocks, and dynamical systems. Brain and cognition, 48(1), 31 51.

Van Kampen, N. G. (1992). Stochastic processes in physics and chemistry (Vol. 1). Elsevier. Wing, A. M. (2002). Voluntary timing and brain function: an information processing approach. Brain and cognition, 48(1), 7-30. 